\title{
Effect of digital image compression on screening for diabetic retinopathy
}

\author{
R S B Newsom, A Clover, M T J Costen, J Sadler, J Newton, A J Luff, C R Canning
}

\begin{abstract}
Backgroundlaims-Digital imaging is widely used for diabetic retinopathy screening. The storage and transmission of digital images can be facilitated by image compression. The authors aimed to assess the effect of image compression on the accuracy of grading diabetic retinopathy.

Methods-Forty nine $35 \mathrm{~mm}$ transparencies (17 with no retinopathy, eight with background, five with preproliferative, and 19 with proliferative retinopathy) were digitised and subjected to JPEG compression by $90 \%, 80 \%, 70 \%$, and $0 \%$. The 196 images were randomised and graded on a portable computer. Two masked graders assessed the images for grade of retinopathy and image quality (0-10). The sensitivity and specificity of retinopathy grading were calculated with a weighted kappa for grading agreement between levels of compression.

Results-The sensitivity of retinopathy grading was reduced by JPEG compression. At $90 \%, 80 \%, 70 \%$, and $0 \%$ compression the sensitivities were $0.38,0.50,0.65$, and 0.72 , respectively; the specificity results were $1.00,1.00,0.83$, and 0.84 , respectively; and the weighted kappa scores were $0.60,0.75,0.77$, and 0.84 , respectively. The quality scores for $90 \%$, $80 \%, 70 \%, 0 \%$ compression were 2.9 (SD 1.1, 95\% CI; 2.7-3.2), 4.6 (SD 1.1, 95\% CI; 3.0-5.6), 5.8 (SD1.5, 95\% CI 5.0-6.6), 6.3 (SD1.4, 95\% CI; 5.4-7.2) $(\mathrm{p}<0.01$ for each intergroup comparison).

Conclusion-The results demonstrate significant loss of sensitivity to the features of diabetic retinopathy with JPEG compression; this was compounded by the thin film transistor (TFT) screen. The authors found the quality of uncompressed images on TFT screens too poor to give grading sensitivities which reach current guidelines for diabetic retinopathy screening. (Br f Ophthalmol 2001;85:799-802)
\end{abstract}

The ease and flexibility of digital photography has led to the widespread use of this technology for ophthalmic imaging, particularly for retinal screening. ${ }^{1-5}$ Several centres now use digital photography for diabetic screening and some have introduced routine digital photography to monitor clinic patients. ${ }^{3}$ Digital photography generally does not give as good an image as conventional $35 \mathrm{~mm}$ photography. However, several authors have found good agreement between both types of image for diabetic retinopathy grading. ${ }^{6}$ Colour digital photography detects diabetic retinopathy $71-$ $85 \%$ of the time, and similar sensitivities have been found with digitised $35 \mathrm{~mm}$ slides. ${ }^{467}$ Sensitivity to sight threatening diabetic retinopathy may be increased from 0.83 to 0.94 using digital enhancement and "neural networks" have been used to detect both normal anatomy and diabetic retinopathy. ${ }^{389}$ Spencer et al have developed the automated the detection of microaneurysms. ${ }^{1011}$

To obtain good quality digital images high density pixelation is necessary; this generates large file sizes, which are expensive to store and difficult to access (on a computer network) or transmit via a modem. ${ }^{12-14}$ Compression reduces the image file size permitting rapid transmission and saving storage space. Compression algorithms are termed "lossy" if they cause a permanent reduction in the information carried by the image and "loss less" if all the initial data are preserved. The Joint Photographic Experts Group (JPEG) is a standard lossy compression used in medical imaging systems and internet image transmission. JPEG compression transforms colours to a luminance/chrominance colour map, pixels are grouped into $8 \times 8$ blocks, averaged, and part of the chrominance component discarded. The amount of compression may be varied from $0-100 \%$, by discarding various amounts of digital information. Other classes of image compression include wavelet compression, which involves image decomposition into signals that vary in scale and orientation, and fractal compression, in which fractals within the image are compressed. ${ }^{15}$

JPEG compression is claimed to allow data compression by $40: 1$, without loss of significant image quality. ${ }^{14}$ However, some suggest there is information loss at the higher compression levels. ${ }^{15}$ Several authors have looked at wavelet versus JPEG compression with little agreement about which is better. ${ }^{15}{ }^{16}$ Compression algorithms are more effective on certain types of images than on others. There is little specific evidence on the effect of JPEG compression on the quality of retinal images, and particularly the effect of compression on screening performance.

The type of display may also affect the detection of diabetic retinopathy. Modern liquid crystal displays (LCD) are based on a thin film transistor (TFT). Screens are made up of a matrix of red, blue, and yellow pixels, illuminated by a back light. The amount of light passing through pixels is determined by the orientation of a liquid crystal controlled by a TFT. TFT screens give good quality images
Accepted for publication 15 January 2001 
and are now replacing traditional cathode ray tube (CRT) screens, as they are smaller, lighter, and flicker free. TFT screens are now used in some hospital networks for digitised notes and in some screening services for digital photography.

There are few performance standards for these new technologies. ${ }^{17}$ We aimed to assess the effect of JPEG image compression on the diagnosis of diabetic retinopathy, when digital images are viewed on a TFT screen.

\section{Methods}

Forty nine $35 \mathrm{~mm}$ transparencies of eyes (17 with no retinopathy, eight with background, five with preproliferative, and 19 with proliferative retinopathy) were digitised to tagged information file format (TIFF) files using the Umax "Powerlook" 3000 scanner at a resolution of 3000 dots per inch (dpi). The images were converted into JPEG files at $0 \%, 70 \%, 80 \%$, and $90 \%$ compression using "Paintshop Pro" and were placed in a random order.

IMAGE GRADING

The images $(n=196)$ were presented in a random order on a 14 inch, 24 bit colour, super extended graphics array (SVGA), thin film transistor (TFT) flat screen $(1024 \times 768$ pixels, $0.28 \mathrm{~mm}$ dot pitch). Two masked graders (both retinal specialists) were presented the images independently under uniform lighting conditions. Images were graded into no retinopathy, background, preproliferative, and proliferative retinopathy based on European grading protocol. ${ }^{18}$ Each grade of retinopathy was considered, as accurate diagnosis of each grade was thought to have significant management implications within a screening programme. Where graders disagreed the images were regraded by a third grader. This process produced a grade for each image at each of the four compression levels (for example, $0 \%$, $70 \%, 80 \%$, and $90 \%$ ).

To create a gold standard the original 49,35 $\mathrm{mm}$ slides were projected to a diameter of 1.4 $\mu \mathrm{m}(\times 4300)$; these were viewed by the two original graders and two further retinal specialists, a consensus grade for each image was reached. To reduce observer bias the gold standard was created 3 months after the original grading.

The grade for each image at each compression level (for example, $0 \%, 70 \%, 80 \%$, and $90 \%$ ) was compared with the gold standard and the specificity, sensitivity, positive predictive value (PPV), and negative predictive value (NPV) were calculated for each level of compression. A weighted kappa statistic of each compression grade was calculated to assess the level of agreement between the gold standard and the grading scores from each level of compression. The kappa approach allows for the level of agreement expected by chance and the weighted kappa statistic takes into account the extent of disagreement when there are more than two categories.
IMAGE QUALITY

The quality of each image was also assessed during grading. The graders were asked to assess the quality of each of the 196 images presented on an analogue score of 0 (worst) to 10 (best). The quality scores from each observer were combined to give an aggregate score for each image. The scores for each level of image compression $(0 \%, 70 \%, 80 \%$, and $90 \%)$ were average, the standard deviation and $95 \%$ confidence interval calculated, and the normality of distribution tested.

The quality score of each image was compared between the groups using the paired $t$ test and the Bonferroni correction.

\section{Results}

IMAGE COMPRESSION DATA

JPEG was an efficient method for image compression. The average file size for $0 \%, 70 \%$, $80 \%$, and $90 \%$ was $4686,133,100$, and 78 kilobytes respectively, with compression ratios of $1: 1,35: 1,46: 1$, and $60: 1$. Figure 1 shows the typical results of $90 \%$ JPEG image compression and an uncompressed image for comparison.

GRADING RESULTS

The screening performance at different compression levels is summarised in Table 1. As expected there was a progressive loss of sensitivity, from 0.72 with no compression to
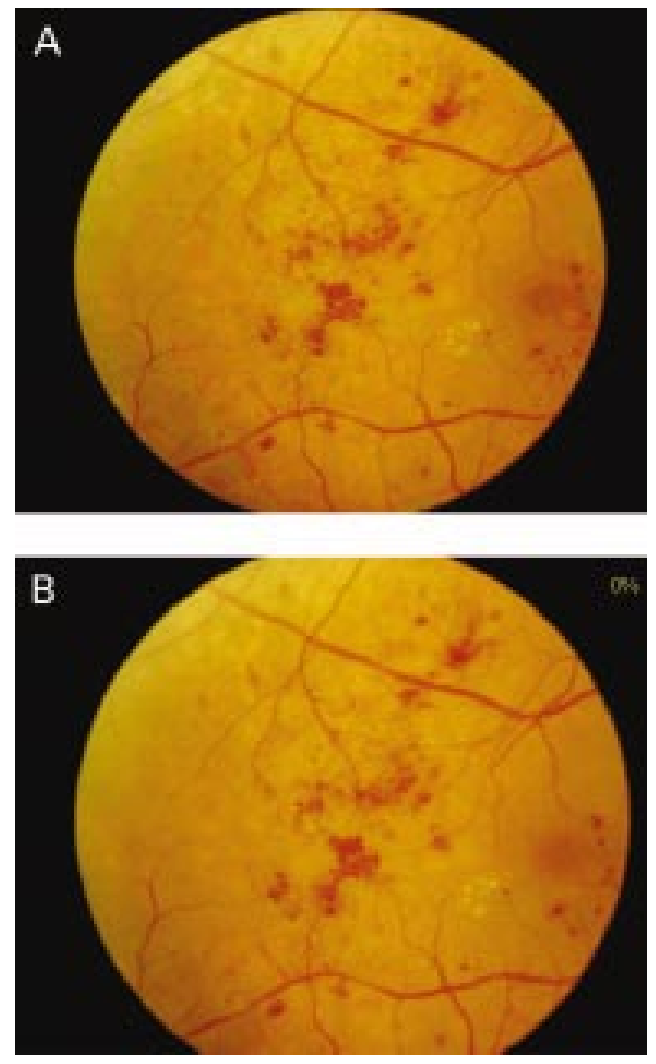

Figure 1 Image compression showing the effect of image compression on an image with proliferative retinopathy. (A) 90\% compression: note colour blocking, increased granularity, with loss of intraretinal microvascular abnormalities, microaneurysms, and neovascularisation. (B) $0 \%$ compression: showing good retinal detail, fine details clear on image. 
Table 1 Screening data for each degree of compression. Note low sensitivities for the compressed images rising to 0.72 for the $0 \%$ compressed image

\begin{tabular}{lllll}
\hline \multicolumn{5}{c}{ Percentage compression } \\
\cline { 2 - 5 } & $90 \%$ & $80 \%$ & $70 \%$ & $0 \%$ \\
\hline Sensitivity & 0.38 & 0.50 & 0.65 & 0.72 \\
Specificity & 1.00 & 1.00 & 0.83 & 0.84 \\
PPV & 1.00 & 1.00 & 0.87 & 0.88 \\
NPV & 0.46 & 0.52 & 0.58 & 0.67 \\
Weighted kappa & 0.60 & 0.75 & 0.77 & 0.84 \\
\hline
\end{tabular}

$\mathrm{PPV}=$ positive predictive value $\mathrm{NPV}=$ negative predictive value.

Table 2 Frequency and extent of misgrading of 49 digital images of diabetic retinopathy with fPEG compression

\begin{tabular}{lrrrr}
\hline & \multicolumn{4}{c}{ Degree of $\mathcal{3}$ PEG compression } \\
\cline { 2 - 5 } Degree of error (grades) & $90 \%$ & $80 \%$ & $70 \%$ & $0 \%$ \\
\hline+1 & & & 3 & 2 \\
0 & 29 & 33 & 35 & 39 \\
-1 & 15 & 15 & 9 & 7 \\
-2 & 2 & & 2 & 1 \\
-3 & 3 & 1 & & \\
\hline
\end{tabular}

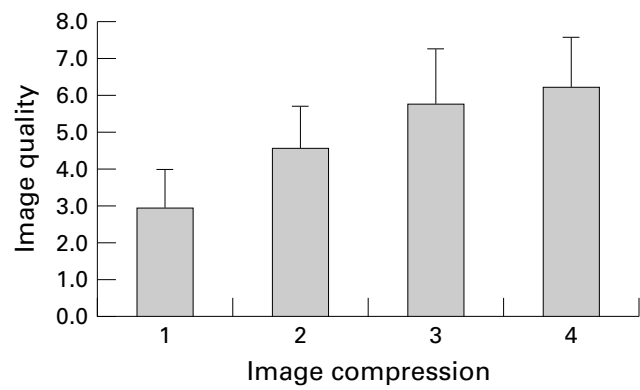

Figure 2 Change in image quality with degree of image compression ( $p<0.01$ for each intergroup comparison).

0.38 with $90 \%$ compression; however, specificity improved from 0.84 with no compression to 1.00 at $90 \%$ compression. Weighted kappa scores were $0.60,0.75,0.77$, and 0.84 for the $90 \%, 80 \%, 70 \%$, and $0 \%$ compressed images confirming a substantial and progressive loss of overall performance as a result of image compression. Table 2 shows that misgrades were both more common and more extreme with greater levels of compression.

IMAGE QUALITY RESULTS

The subjective image quality was reduced by compression (Fig 2). The average quality score (from 1-10), for $90 \%, 80 \%, 70 \%, 0 \%$ compression were 2.9 (SD 1.1; 95\% CI 2.7-3.2), 4.6 (SD 1.1; 95\% CI 3.0-5.6), 5.8 (SD 1.5; 95\% CI 5.0-6.6), and 6.3 (SD1.4; 95\% CI 5.4-7.2) respectively. The data were normally distributed, the quality of each group was found to be significantly different $(\mathrm{p}<0.01$; paired $t$ test, Bonferroni method).

\section{Discussion}

Image compression significantly reduced sensitivity of grading from 0.72 for no compression to 0.38 for $90 \%$ compression. This was an unexpected finding as initially the compressed images appeared to be of excellent quality. The score for the $0 \%$ compression image was lower than that of $0.85-0.95$ previously reported for digital images. ${ }^{78}$
The low sensitivities were reflected in poor image quality assessments (2.9-5.8) for the compressed images. From these data we felt that TFT monitors do not give sufficient image quality to grade accurately. There are few data on the use of TFT screens for grading diabetic retinopathy and although they are generally brighter than CRT screens $\left(215 \mathrm{~cd} / \mathrm{m}^{2}\right.$ versus $100 \mathrm{~cd} / \mathrm{m}^{2}$ ) they have a lower contrast ratio (300:1 $v 550: 1)$, a lower dot pitch (0.28 $v 0.22)$, and poorer colour tuning for the human eye (gamma). CRT screens are generally superior for fine detail and colour or contrast dependent applications such as diabetic retinopathy screening. These drawbacks may be ameliorated using digital enlargement; however, CRT screens still have significant advantages for displaying images of diabetic retinopathy. Despite the moderate image quality graders were extremely sensitive to image compression, and reliably separated $70 \%$ from $80 \%$ compression. Image compression for medical images is still under investigation. ${ }^{151619} \mathrm{~A}$ recent study showed wavelet was superior to JPEG and fractal compression in preserving low contrast details on radiographs. ${ }^{15}$ Others have found that wavelet compression yielded minimally better compression quality at similar levels of compression. ${ }^{16}$ Lazzario et al have developed an image transcoder that enhances JPEG decoding which makes allowances for information loss. Others have been developing new lossless algorithm "PEG-LS" which preserves all digital information during compression. ${ }^{19} 20$ These and other similar developments may allow compression with no loss of clinical information.

Previous research has noted that the amount of compression is related to the type of file captured. Most of the work has been done on radiographs and there is little information on the effect of compression of retinal images, which are predominantly red shades. However, our results do demonstrate that compression ratios over 30:1 with JPEG compression do lead to a significant loss of image quality.

The development of any new technology is not without setbacks. In this paper we have shown that screening for diabetic retinopathy using compressed images on a TFT screen is not sensitive enough to reach standards set by the British Diabetic Association. ${ }^{21}$ We have also shown graders will note both a reduction in image quality and sensitivity when assessing JPEG compressed images on a TFT screen. New methods of image compression may reduce the amount of image distortion following compression and new flat screen monitors may produce clearer images. Until such a time all digital retinal images should be uncompressed and viewed on a CRT monitor.

1 Yogesan K, Constable I, Eikelboom R, et al. Tele-ophthalmic screening using digital imaging devices. Aust NZ F Ophthalmol 1988;26:s9-11.

2 Kerr D, Cavan D, Jennings B, et al. Beyond retinal screening: digital imaging in the assessment and follow-up of patients with diabetic retinopathy. Diabet Med 1998:15: 878-82.

3 Gardner G, Keating D, Williamson T, et al. Automatic detection of diabetic retinopathy using an artificial neural 1996;80:940-4.

Ryder R, Kong N, Bates A, et al. Instant electronic imaging systems are superior to Polaroid at detecting sightthreatening diabetic retinopathy. Diabet Med 1998;15:254-8. 
5 Taylor D, Fisher J, Jacob J, et al. The use of digital cameras in a mobile retinal screening environment. Diabet Med in a mobile re

6 George L, Leverton C, Young S, et al. Can digitised colour $35 \mathrm{~mm}$ transparencies be used to diagnose diabetic retinopathy? Diabet Med 1997;14:970-3.

7 George L, Halliwell M, Hill R, et al. A comparison of digital retinal images and $35 \mathrm{~mm}$ colour transparencies in detecting and grading diabetic retinopathy. Diabet Med 1988;15 $250-3$.

8 George L, Lusty J, Owens $\mathrm{D}$, et al. Effect of software manipulation (Photoshop) of digitised retinal images on the gradin 0

9 Sinthanayothin C, Boyce J, Cook H, et al. Automated localisation of the optic disc, fovea, and retinal blood vessels from digital colour fundus images. $\mathrm{Br} \mathcal{F}$ Ophthalmol 1999;83:902-10.

10 Spencer T, Phillips R, Sharp P, et al. Automated detection and quantification of microaneurysms in fluorescein and quantification of microaneurysms in

11 Cree M, Olson J, McHardy K, et al. A fully automated comparative microaneurysm digital detection system. Eye parative micro

12 Jensen P, Scherfig E. Resolution of retinal digital colour images. Acta Ophthalmol Scand 1999;5:526-9.

13 Schwartz S, Harrison S, Ferrone P, et al. World wide web interface to digital imaging and communication inmedicine-capable image servers.
14 Telemedical evaluation and management of retinopathy of prematurity using a fiberoptic digital fundus camera. $\mathcal{F}$ Digit Imaging 1996;9:178-84.

15 Oka A, Harima Y, Nakano Y, et al. Inter-hospital network system using the worldwide web and the common gateway interface. F Digit Imaging 1999;12:205-7.

16 Ricke J, Maass P, Lopez Hanninen E, et al. Wavelet versus JPEG (Joint Photographic Expert Group) and fractal compression. Impact on the detection of low-contrast details in pression. Impact on the detection of low-contrast details
computed radiographs. Invest Radiol 1998;33:456-63.

17 Iyriboz T, Zukoski M, Hopper K, et al. A comparison of wavelet and Joint Photographic Experts Group lossy compression methods applied to medical images. $\mathcal{F}$ Digit Imaging 1999;12:14-7.

18 Lee P. Telemedicine: opportunities and challenges for the remote care of diabetic retinopathy. Arch Ophthalmol 1999; 117:1639-40.

19 Party RW. A protocol for screening for diabetic retinopathy in Europe. Diab Med 1991;8:263-7.

20 Kivijarvi J, Ojala T, Kaukoranta T, et al. A comparison of lossless compression methods for medical images. Comput Med Imaging Graph 1988;22:323-39.

21 Lazzaro J, Wawrzynek J. JPEG quality transcoding using neural networks trained with a perceptual error measure. Neural Comput 1999;11:267-96.

22 British Diabetic Association. Guidelines on screening for diabetic retinopathy. London: BDA, 1999. 